\title{
Upper bounds on wavepacket spreading for random Jacobi matrices
}

\author{
Svetlana Jitomirskaya ${ }^{1}$, Hermann Schulz-Baldes ${ }^{2}$ \\ 1 Department of Mathematics, University of California at Irvine, Ca, 92697, USA \\ 2 Mathematisches Institut, Universität Erlangen-Nürnberg, Germany
}

\begin{abstract}
A method is presented for proving upper bounds on the moments of the position operator when the dynamics of quantum wavepackets is governed by a random (possibly correlated) Jacobi matrix. As an application, one obtains sharp upper bounds on the diffusion exponents for random polymer models, coinciding with the lower bounds obtained in a prior work. The second application is an elementary argument (not using multiscale analysis or the Aizenman-Molchanov method) showing that under the condition of uniformly positive Lyapunov exponents, the moments of the position operator grow at most logarithmically in time.
\end{abstract}

\section{Introduction}

One of the fundamental questions of quantum mechanics concerns the spreading of an initially localized wave packet $\phi$ under the time evolution $e^{-\imath t H}$ associated to a Schrödinger operator $H$. If the physical space is $\mathbb{R}^{d}$ or $\mathbb{Z}^{d}$ and the position operator is denoted by $X$, the spreading can be quantified using the time-averaged moments of $X$ (or equivalently the moments of the associated classical probability distribution):

$$
M_{T}^{q}=\int_{0}^{\infty} \frac{d t}{T / 2} e^{-\frac{2 t}{T}}\left\langle\left.\phi\left|e^{\imath H t}\right| X\right|^{q} e^{-\imath H t} \mid \phi\right\rangle, \quad q>0 .
$$

It is well known that for short-range operators $H$, the moments cannot grow faster than ballistically, that is $M_{T}^{q} \leq C(q) T^{q}$. The growth actually is ballistic in typical scattering situations and for periodic operators $H$ describing Bloch electrons. On the other hand, if the moments $M_{T}^{q}$ are bounded uniformly in time, one speaks of dynamical localization. This can be proven in the regime of Anderson localization for random operators, but also for certain almost-periodic operators (see Jjit for a review). There are many models where the moments $M_{T}^{q}$ exhibit some non-trivial power law behavior. If $M_{T}^{q} \sim T^{q / 2}$, the quantum motion is diffusive, and any 
other asymptotic growth behavior is called anomalous diffusion. In order to distinguish various anomalous diffusive motions, one defines the diffusion exponents

$$
\beta_{q}^{+}=\limsup _{T \rightarrow \infty} \frac{\log \left(M_{T}^{q}\right)}{\log \left(T^{q}\right)}, \quad \beta_{q}^{-}=\liminf _{T \rightarrow \infty} \frac{\log \left(M_{T}^{q}\right)}{\log \left(T^{q}\right)}, \quad q>0 .
$$

If the limit exists, we write $\beta_{q}=\beta_{q}^{+}=\beta_{q}^{-}$. The diffusion exponents correspond to the LevyKhinchin classification of Levy flights in classical probability, however, we stress that the quantum anomalous diffusion does not result from a probabilistic dynamics, but rather from a Hamiltonian one. It is due to delicate quantum interference phenomena. The ballistic bound implies $0 \leq \beta_{q}^{ \pm} \leq 1$ and convexity inequalities show that $\beta_{q}^{ \pm}$is non-decreasing in $q$. In the regime of dynamical localization $\beta_{q}=0$ and for quantum diffusion $\beta_{q}=\frac{1}{2}$. Anomalous diffusion corresponds to all other values of $\beta_{q}^{ \pm}$. Typically $\beta_{q}^{ \pm}$is then also varying with $q$ which reflects a rich multiscale behavior of the wave packet spreading. Such anomalous diffusion was exhibited numerically in several almost-periodic Jacobi matrices having singular continuous spectra (e.g. Fibonacci and critical Harper operator), and also some random and sparse Jacobi matrices.

It is a challenging problem of mathematical physics to calculate the diffusion exponents for a given Schrödinger operator, in particular, when the quantum motion is anomalous diffusive. In this work we accomplish this for the so-called random polymer models, a random Jacobi matrix described in detail in the next section, and show that

$$
\beta_{q}=\max \left\{0,1-\frac{1}{2 q}\right\}, \quad q>0,
$$

see Theorem 3. This result confirms the heuristics and numerical results of Dunlap, Wu and Phillips [DWP] for the random dimer model, the prototype of a the random polymer. The latter model was introduced and analyzed in our prior work in collaboration with G. Stolz [JSS], which already contained a rigorous proof of the lower bound $\beta_{q}^{-} \geq 1-\frac{1}{2 q}$. In this work we hence focus on the upper bound, which amounts to proving quantitative localization estimates.

Next let us discuss this result in the context of prior rigorous work on other one-dimensional models (Jacobi matrices) exhibiting anomalous diffusion. First of all, the Guarneri bound [Gua and its subsequent improvements [Com, Las, GS2, GS3, KL, BGT] allow to estimate the diffusion exponents from below in terms of various fractal dimensions of the spectral measure. However, those results do not allow to prove the lower bound in (3) because, as was shown by de Bievre and Germinet [BG], the random dimer model has pure-point spectrum so that the Hausdorff dimension vanishes and the Guarneri bound is empty. In fact, the argument in [JSS] is based on a large deviation estimate on the localization length of the eigenstates near the so-called critical energies at which the Lyapunov exponent vanishes.

Upper bounds on anomalous quantum diffusion were first proven for Jacobi matrices with self-similar spectra [GS1, BS], and these bounds are even optimal for the so-called Julia matrices. Kiselev, Killip and Last [KKL] presented a technique based on subordinacy theory allowing to control the spread of a certain portion of the wave packet (not the fastest one and therefore not the moments), and applied it to the Fibonacci model. Tcheremchantsev [Tch] proved 
tight upper bounds on growing sparse potential Hamiltonians introduced in [JL] and further analyzed in [CM]. Recently, Damanik and Tcheremchantsev [DT] developed a transfer matrix based method that allows to prove upper bounds on the diffusion exponents and also applied it to the Fibonacci model. Another way to achieve upper bounds on the diffusion exponents in terms of properties of the finite size approximants (Thouless widths and eigenvalue clustering) was recently developed and applied to the Fibonacci operator by Breuer, Last, and Strauss [BLS]. In the models considered in [GS1, BS, DT, BLS, Tch] the anomalous diffusion is closely linked to dimensional properties of the spectral measures, even though the (generalized) eigenfunctions have to be controlled as well. As a result, the transport slows down as the fractal dimension of the spectral measure decreases. The origin of the anomalous transport in the random polymer model is of different nature. In fact, in the random polymer model only a few, but very extended localized states near the critical energies lead to the growth of the moments. Hence this model illustrates that spectral theory may be of little use for the calculation of the diffusion exponents. This statement is even more true if the dimension of physical space is higher. There are examples of three-dimensional operators with absolutely continuous spectral measures, but subdiffusive quantum diffusion with diffusion exponents as low as $\frac{1}{3}[\mathrm{BeS}]$.

The strategy for proving upper bounds advocated in [DT] appears to be more efficient in the present context than prior techniques GS1, KKL. We refine and generalize the relevant part in Section 3, see in particular Proposition 2. It allows to give a rather simple proof of our second result, namely Theorem 1, which establishes a logarithmic bound $M_{T}^{q} \leq \log (T)^{\beta q}, \beta>2$, under the condition of uniformly positive Lyapunov exponent. This result is neither new nor fully optimal. However, in the generality we have (any condition on the distribution of randomness, including e.g. Bernoulli) the Aizenman-Molchanov method [AM] cannot be applied, and the only technique previously available to obtain this statement was the multi-scale analysis of [CKM] (see also [BG, DSS]). Thus our proof is significantly simpler. Moreover, one can argue that it captures the physically relevant effect of localization. Indeed, it was shown by Gordon [Gor] and del Rio, Makarov and Simon [DMS] that a generic rank one perturbation of a model in the regime of strict dynamical localization $\left(M_{T}^{q}\right.$ bounded) leads to singular continuous spectrum, and therefore, by the RAGE theorem, growth of the moments. However, it was shown in DJLS] that this growth can be at most logarithmic, just as proven in Theorem 1 . The proof of Theorem 1 constitutes essentially a part of the proof of Theorem 3 ,

In the next section we present our models and results with technical details. Section 3 contains a general (non-random) strategy for proving the upper bounds. Section 4 provides the proof of Theorem 11 as well as some statements that are used in Section 6. In Section 5 we obtain probabilistic bounds on the transfer matrices near a critical energy based on the large deviation estimate of [JSS]. Section 6 contains the proof of Theorem 3, that is the identity (3).

Acknowledgment: This work would have been impossible without [JSS]. We are very thankful to G. Stolz for this collaboration. We also thank the anonymous referees for comments that improved the paper. The work of S. J. was supported in part by the NSF, grant DMS-0300974, and Grant No. 2002068 from the United States-Israel Binational Science Foundation (BSF), Jerusalem, Israel. H. S.-B. acknowledges support by the DFG. 


\section{Models and results}

A Jacobi matrix is an operator $H_{\omega}$ on $\ell^{2}(\mathbb{Z})$ associated to the data $\omega=\left(t_{n}, v_{n}\right)_{n \in \mathbb{Z}}$ of positive numbers $t_{n}$ and real numbers $v_{n}$ which we suppose to be both bounded by a constant $C$, and $t_{n}$ bounded away from 0 . Using the Dirac notation $|n\rangle$ for the canonical basis in $\ell^{2}(\mathbb{Z}), H_{\omega}$ is given by

$$
H_{\omega}|n\rangle=t_{n+1}|n+1\rangle+v_{n}|n\rangle+t_{n}|n-1\rangle .
$$

Each $\omega$ is called a configuration. The set of all configurations is contained in $\Omega=\left([-C, C]^{\times 2}\right)^{\times \mathbb{Z}}$. The left shift $S$ is naturally defined on $\Omega$. A stochastic Jacobi matrix is a family $\left(H_{\omega}\right)_{\omega \in \Omega}$ of Jacobi matrices drawn according to a probability measure $\mathbf{P}$ on $\Omega$ which is invariant and ergodic w.r.t. to $S$. Furthermore, we speak of a random Jacobi matrix if $\mathbf{P}$ has at most finite distance correlations, namely there exists a finite correlation length $L \in \mathbb{N}$ such that $\left(t_{n}, v_{n}\right)$ and $\left(t_{m}, v_{m}\right)$ are independent whenever $|n-m| \geq L$. Most prominent example of a random Jacobi matrix is the one-dimensional Anderson model for which $t_{n}=1$ and the $v_{n}$ are independent and identically distributed so that $L=1$. Random polymer models as studied in [DWP, JSS] and described in more detail below provide an example of a random Jacobi matrix with finite distance correlations (the second crucial feature of these models is that the $\left(t_{n}, v_{n}\right)$ only take a finite number of values).

We will consider the disorder and time averaged moments of the position operator $X$ on $\ell^{2}(\mathbb{Z})$, denoted by $M_{T}^{q}$ as in (11):

$$
M_{T}^{q}=\int_{0}^{\infty} \frac{d t}{T / 2} e^{-\frac{2 t}{T}} \mathbf{E}\left\langle\left. 0\left|e^{\imath H_{\omega} t}\right| X\right|^{q} e^{-\imath H_{\omega} t} \mid 0\right\rangle, \quad q>0 .
$$

Here $\mathbf{E}$ denotes the average over $\omega$ w.r.t. P. (Note that upper bounds on the expectation w.r.t. $\mathbf{P}$ yield upper bounds almost surely.) One may replace $|0\rangle$ by any other localized initial state (at least in $\ell^{1}(\mathbb{Z})$ ).

As discussed in the introduction, it is well-known that the Anderson model exhibits dynamical localization, that is $M_{T}^{q} \leq C(q)<\infty$ uniformly in $T$ for all $q>0$. A by-product of our analysis of the random polymer model discussed below is a simple proof of the weaker result that $M_{T}^{q}$ grows at most logarithmically in $T$ whenever the Lyapunov exponent is strictly positive. In order to define the latter, let us introduce as usual in the analysis of one-dimensional systems the transfer matrices at a complex energy $z$ by

$$
\mathcal{T}_{\omega}^{z}(n, m)=\mathcal{T}_{n-1}^{z} \cdot \ldots \cdot \mathcal{T}_{m}^{z}, \quad n>m, \quad \mathcal{T}_{n}^{z}=\left(\begin{array}{cc}
\left(z-v_{n}\right) t_{n}^{-1} & -t_{n} \\
t_{n}^{-1} & 0
\end{array}\right) .
$$

Furthermore $\mathcal{T}_{\omega}^{z}(n, m)=\mathcal{T}_{\omega}^{z}(m, n)^{-1}$ for $n<m$ and $\mathcal{T}_{\omega}^{z}(n, n)=\mathbf{1}$. Then the Lyapunov exponent is

$$
\gamma(z)=\lim _{N \rightarrow \infty} \frac{1}{N} \mathbf{E} \log \left(\left\|\mathcal{T}_{\omega}^{z}(N, 0)\right\|\right)
$$

Because the $t_{n}$ and $v_{n}$ are uniformly bounded, one shows by estimating the norm of a product of matrices by the product of their norms that for every bounded set $U \subset \mathbb{C}$

$$
\left\|\mathcal{T}_{\omega}^{z}(n, m)\right\| \leq e^{\gamma_{1}|n-m|}, \quad z \in U
$$


where the $\gamma_{1}$ depends on $U$. This implies that $\gamma(z) \leq \gamma_{1}$ for $z \in U$. For a random Jacobi matrix, uniform lower bounds on $\gamma(z)$ can be proven by the Furstenberg theorem (e.g. [PF]). This applies in particular to the Anderson model (also with a Bernoulli potential), and more generally to random Jacobi matrices with correlation length equal to 1.

Theorem 1 Consider a random Jacobi matrix. Let the (non-random) spectrum be $\sigma(H) \subset$ $\left(E_{0}, E_{1}\right)$ Suppose that the Lyapunov exponent is strictly positive:

$$
\gamma(z) \geq \gamma_{0}>0, \quad z \in\left(E_{0}, E_{1}\right)
$$

Then for any $\beta>2$ there exists a constant $C(\beta, q)$ such that

$$
M_{T}^{q} \leq(\log T)^{q \beta}+C(\beta, q) .
$$

Another self-averaging quantity associated to to ergodic Jacobi matrices is the integrated density of states (IDS) $\mathcal{N}(E)$ which can be defined by

$$
\int \mathcal{N}(d E) f(E)=\mathbf{E}\left\langle 0\left|f\left(H_{\omega}\right)\right| 0\right\rangle, \quad f \in C_{0}(\mathbb{R}) .
$$

The Thouless formula (e.g. $[\mathrm{PF}]$ ) links the Lyapunov exponent to the IDS. It implies that, if (17) holds for real $E \in\left(E_{0}, E_{1}\right)$, then one also has $\gamma(z) \geq \gamma_{0}$ for all $z$ with $\Re e(z) \in\left(E_{0}, E_{1}\right)$.

As shown by Theorem 1 and the remark just before it, it is necessary for a random Jacobi matrix to have a correlation length larger than 1 for the moments $M_{T}^{q}$ to grow faster than logarithmically, so that the diffusion exponents $\beta_{q}$ do not vanish. That this actually happens for the random dimer model was discovered by Dunlap, Wu and Phillips [DWP].

Next let us describe in more detail the more general random polymer model considered in [JSS]. Given are two finite sequences $\hat{t}_{ \pm}=\left(\hat{t}_{ \pm}(0), \ldots, \hat{t}_{ \pm}\left(L_{ \pm}-1\right)\right)$ and $\hat{v}_{ \pm}=\left(\hat{v}_{ \pm}(0), \ldots, \hat{v}_{ \pm}\left(L_{ \pm}-\right.\right.$ $1)$ ) of real numbers, satisfying $\hat{t}_{ \pm}(l)>0$ for all $l=0, \ldots, L_{ \pm}-1, L_{ \pm} \geq 1$. The associated random polymer model is the random Jacobi matrix constructed by random juxtaposition of these sequences and randomizing the origin. More precisely, configurations $\omega \in \Omega$ can be identified with the data of a sequence of signs $\left(\sigma_{n}\right)_{n \in \mathbb{Z}}$ and an integer $0 \leq l \geq L_{\sigma_{1}}-1$, via the correspondence $\left(t_{n}\right)=\left(\ldots, \hat{t}_{\sigma_{1}}(l), \ldots, \hat{t}_{\sigma_{1}}\left(L_{\sigma_{1}}-1\right), \hat{t}_{\sigma_{2}}(0), \ldots, \hat{t}_{\sigma_{2}}\left(L_{\sigma_{2}}-1\right), \hat{t}_{\sigma_{3}}(0), \ldots\right)$ and similarly for $\left(v_{n}\right)$, with choice of origin $t_{0}=\hat{t}_{\sigma_{1}}(l)$ and $v_{0}=\hat{v}_{\sigma_{1}}(l)$. The shift is as usual, and the probability $\mathbf{P}$ is the Bernoulli measure with probabilities $p_{+}$and $p_{-}=1-p_{+}$combined with a randomization for $l$ ( $c f$. [JSS] for details). The correlation length in this model is $L=\max \left\{L_{+}, L_{-}\right\}$. It is now natural and convenient to consider the polymer transfer matrices

$$
T_{ \pm}^{z}=\mathcal{T}_{\hat{v}_{ \pm}\left(L_{ \pm}-1\right), \hat{t}_{ \pm}\left(L_{ \pm}-1\right)}^{z} \cdot \cdots \cdot \mathcal{T}_{\hat{v}_{ \pm}(0), \hat{t}_{ \pm}(0)}^{z}, \quad \text { where } \quad \mathcal{T}_{v, t}^{z}=\left(\begin{array}{cc}
(E-v) t^{-1} & -t \\
t^{-1} & 0
\end{array}\right) .
$$

Definition 1 An energy $E_{c} \in \mathbb{R}$ is called critical for the random polymer model $\left(H_{\omega}\right)_{\omega \in \Omega}$ if the polymer transfer matrices $T_{ \pm}^{E_{c}}$ are elliptic (i.e. $\left|\operatorname{Tr}\left(T_{ \pm}^{E_{c}}\right)\right|<2$ ) or equal to $\pm \mathbf{1}$ and commute

$$
\left[T_{-}^{E_{c}}, T_{+}^{E_{c}}\right]=0
$$


If $L_{ \pm}=1$, the model reduces to the Bernoulli-Anderson model and there are no critical energies. The most studied [DWP, Bov, $\mathrm{BG}$ ] example is the random dimer model for which $L_{+}=L_{-}=2$ and $\hat{t}_{ \pm}(0)=\hat{t}_{ \pm}(1)=1, \hat{v}_{+}(0)=\hat{v}_{+}(1)=\lambda$ and $\hat{v}_{-}(0)=\hat{v}_{-}(1)=-\lambda$ for some $\lambda \in \mathbb{R}$. This model has two critical energies $E_{c}=\lambda$ and $E_{c}=-\lambda$ as long as $\lambda<1$. For further examples we refer to [JSS]. It follows from the definition that a simultaneous change of coordinates reduces both $T_{+}$and $T_{-}$to rotations by angles that are denoted by $\eta_{+}$and $\eta_{-}$.

It is immediate from (10) that the Lyapunov exponent vanishes at a critical energy. Because the transfer matrices $T_{ \pm}^{z}$ are analytic in $z$, it follows that there is a constant $c_{0}$ such that for all $\epsilon \in \mathbb{C}$ with $|\epsilon|<\epsilon_{0}$ one has for $n, m \in \mathbb{N}$,

$$
\left\|\mathcal{T}_{\omega}^{E_{c}+\epsilon}(n, m)\right\| \leq e^{c_{0}|\epsilon| n-m \mid} .
$$

In particular, $\left|\gamma\left(E_{c}+\epsilon\right)\right| \leq c_{0}|\epsilon|$. However, the correct asymptotics for the Lyapunov exponent is $\gamma\left(E_{c}+\epsilon\right)=\mathcal{O}\left(\epsilon^{2}\right)$. This was first shown (non-rigorously) by Bovier for the case of the random dimer model $\mathrm{Bov}$, but heuristics were already given in [DWP]. The rigorous result about the Lyapunov exponent and also the integrated density of states $\mathcal{N}$ are combined in the following theorem.

Theorem 2 [JSS Suppose that $\mathbf{E}\left(e^{2 \imath \eta_{\sigma}}\right) \neq 1$ and $\mathbf{E}\left(e^{4 \imath \eta_{\sigma}}\right) \neq 1$. Then for $\epsilon \in \mathbb{R}$ and some $D \geq 0$, the Lyapunov exponent of a random polymer model satisfies

$$
\gamma\left(E_{c}+\epsilon\right)=D \epsilon^{2}+\mathcal{O}\left(\epsilon^{3}\right),
$$

in the vicinity of a critical energy $E_{c}$. If $\left\|\left[T_{-}^{E_{c}+\epsilon}, T_{+}^{E_{c}+\epsilon}\right]\right\| \geq C \epsilon$ for some $C>0$ and small $\epsilon$, one has $D>0$. Moreover, the IDS $\mathcal{N}$ satisfies

$$
\mathcal{N}\left(E_{c}+\epsilon\right)-\mathcal{N}\left(E_{c}-\epsilon\right)=D^{\prime} \epsilon+\mathcal{O}\left(\epsilon^{2}\right),
$$

for some constant $D^{\prime}>0$.

Furthermore [JSS contains explicit formulas for $D$ and $D^{\prime}$. The bound $D>0$ is not explicitely contained in [JSS], but can be efficiently checked using Proposition 1 in [SSS]. The statement about the IDS only requires $\mathbf{E}\left(e^{2 \imath \eta_{\sigma}}\right) \neq 1$. Let us remark that the hypothesis $\mathbf{E}\left(e^{4 \imath \eta_{\sigma}}\right) \neq 1$ does not hold, for example, in the special case of a random dimer model if $\lambda=1 / \sqrt{2}$. In this situation, one is confronted with an anomaly. Nevertheless, the asymptotics is as in (12) and again one can calculate $D$ explicitely [Sch]. As we did not perform the large deviation analysis of [JSS] in the case of an anomaly, we retain the hypothesis of Theorem 2 throughout.

The following is the main result of this work.

Theorem 3 Suppose that $\mathbf{E}\left(e^{22 \eta_{\sigma}}\right) \neq 1$ and $\mathbf{E}\left(e^{4 \imath \eta_{\sigma}}\right) \neq 1$, and that the random polymer model has a critical energy at which (12) holds with $D>0$. Then for $q>0$,

$$
\beta_{q}=\max \left\{0,1-\frac{1}{2 q}\right\} .
$$

As already pointed out, the lower bound $\beta_{q}^{-} \geq 1-\frac{1}{2 q}$ was already proven in [JSS]. 


\section{A strategy for proving upper bounds on dynamics}

In this section it is not necessary for the Jacobi matrix to be random or ergodic; hence the index $\omega$ and the average E are suppressed. Let the notation for the Green's function be

$$
G^{z}(n, m)=\left\langle n\left|\frac{1}{H-z}\right| m\right\rangle
$$

where $n, m \in \mathbb{Z}$ and $z \in \mathbb{C}$ is not in the spectrum $\sigma(H)$ of $H$. The starting point of the analysis is to express the time averaged moments (5) in terms of the Green's function

$$
M_{T}^{q}=\sum_{n \in \mathbb{Z}}|n|^{q} \int_{\mathbb{R}} \frac{d E}{\pi T}\left|G^{E+\frac{\imath}{T}}(0, n)\right|^{2} .
$$

Using the spectral theorem, this well known identity can be checked immediately by a contour integration. In order to decompose the expression on the r.h.s., let us introduce for $0<\alpha_{0}<\alpha_{1}$ and $E_{0} \leq E_{1}$,

$$
M_{T}^{q, \alpha_{0}, \alpha_{1}}\left(E_{0}, E_{1}\right)=\sum_{T^{\alpha_{0}}<|n| \leq T^{\alpha_{1}}}|n|^{q} \int_{E_{0}}^{E_{1}} \frac{d E}{\pi T}\left|G^{E+\frac{\imath}{T}}(0, n)\right|^{2},
$$

and $M_{T}^{q, 0, \alpha}\left(E_{0}, E_{1}\right)$ is defined similarly with the sum running over $0 \leq|n| \leq T^{\alpha}, \alpha>0$. The following result also holds for higher dimensional models.

Proposition 1 Suppose $\sigma(H) \subset\left(E_{0}, E_{1}\right)$ and $\alpha>1$. Set $\Delta=\operatorname{dist}\left(\left\{E_{0}, E_{1}\right\}, \sigma(H)\right)$. Then there exists a constant $C_{1}=C_{1}(\alpha, \Delta, q)$ such that

$$
\left|M_{T}^{q}-M_{T}^{q, 0, \alpha}\left(E_{0}, E_{1}\right)\right| \leq \frac{C_{1}}{T} .
$$

The proof is based on the following Combes-Thomas estimate. Even though standard, its proof is sufficiently short and beautiful to reproduce it.

Lemma 1 Let $\Delta(z)=\operatorname{dist}(z, \sigma(H))$ and $C_{2}=\left(4\|t\|_{\infty}\right)^{-1}$ where $\|t\|_{\infty}=\sup _{n \in \mathbb{Z}} t_{n}$. Then

$$
\left|G^{z}(n, m)\right| \leq \frac{2}{\Delta(z)} \exp \left(-\operatorname{arcsinh}\left(C_{2} \Delta(z)\right)|n-m|\right) .
$$

Proof. For $\eta \in \mathbb{R}$, set $H_{\eta}=e^{\eta X} H e^{-\eta X}$. A short calculation shows that

$$
\left\|H_{\eta}-H\right\| \leq\|t\|_{\infty}\left|e^{\eta}-e^{-\eta}\right|
$$

Hence one has

$$
\left\|\left(H_{\eta}-z\right)^{-1}\right\| \leq\left(\left\|(H-z)^{-1}\right\|^{-1}-\left\|H_{\eta}-H\right\|\right)^{-1}
$$

Since $\left\|(H-z)^{-1}\right\| \leq \Delta(z)^{-1}$, the choice $|\eta|=\operatorname{arcsinh}\left(\Delta(z) /\left(4\|t\|_{\infty}\right)\right)$ hence gives $\|\left(H_{\eta}-\right.$ $z)^{-1} \| \leq 2 / \Delta(z)$. The bound now follows from $\left\langle n\left|(H-z)^{-1}\right| m\right\rangle=e^{\eta(m-n)}\left\langle n\left|\left(H_{\eta}-z\right)^{-1}\right| m\right\rangle$.

The following estimate will be used not only for the proof of Proposition 1, but at several reprises below. 
Lemma 2 Let $\Delta, \alpha>0, q \geq 0$ and $N \in \mathbb{N}$. Let $p=\left[\frac{q+1}{\alpha}\right]$ where $[\cdot]$ stands for the integer part. Then

$$
\sum_{n>N} n^{q} e^{-\Delta n^{\alpha}} \leq \frac{2}{\alpha} p !\left(N+\Delta^{-1}\right)^{p} \frac{e^{-\Delta N^{\alpha}}}{\Delta}
$$

Proof. Bounding the sum by the integral on each interval of monotonicity and then extending this integral to the entire range $[N, \infty)$, we obtain

$$
\sum_{n>N} n^{q} e^{-\Delta n^{\alpha}} \leq 2 \int_{N}^{\infty} d x x^{q} e^{-\Delta x^{\alpha}} \leq \frac{1}{\alpha} \int_{N^{\alpha}}^{\infty} d y y^{p} e^{-\Delta y}=\frac{e^{-\Delta N^{\alpha}}}{\alpha \Delta^{1+p}} \sum_{j=0}^{p} \frac{p !}{j !}(N \Delta)^{j} .
$$

Bounding the sum over $j$ by $p !(N \Delta+1)^{p}$ completes the proof.

Proof of Proposition [1. We first consider the energies above the spectrum and set $\Delta=$ $\operatorname{dist}\left(E_{1}, \sigma(H)\right)$. Due to the previous two lemmata and if $p=[q+1]$,

$$
\begin{aligned}
M_{T}^{q, 0, \infty}\left(E_{1}, \infty\right) & \leq \int_{0}^{\infty} \frac{d E}{\pi T} \sum_{|n| \geq 1}|n|^{q} \frac{2}{\Delta+E} \exp \left(-\operatorname{arcsinh}\left(C_{2}(\Delta+E)\right)|n|\right) \\
& \leq \int_{0}^{\infty} \frac{d E}{\pi T} \frac{8 p !}{\Delta+E} \operatorname{arcsinh}\left(C_{2}(\Delta+E)\right)^{-(p+1)} .
\end{aligned}
$$

Since $\operatorname{arcsinh}(y) \geq \ln (y)$ for large $y$, this shows that $M_{T}^{q, 0, \infty}\left(E_{1}, \infty\right) \leq C / T$ for some constant $C=C(q, \Delta)$. A similar bound holds for $M_{T}^{q, 0, \infty}\left(-\infty, E_{0}\right)$. Now using the imaginary part of the energy in Lemma 1 and the bound $\operatorname{arcsinh}(y) \geq y$ for sufficiently small $y \geq 0$, we obtain

$$
\begin{aligned}
M_{T}^{q, \alpha, \infty}\left(E_{0}, E_{1}\right) & \leq \frac{2}{\pi} \int_{E_{0}}^{E_{1}} d E \sum_{|n|>T^{\alpha}}|n|^{q} \exp \left(-\operatorname{arcsinh}\left(C_{2} / T\right)|n|\right) \\
& \leq \frac{4\left(E_{1}-E_{0}\right) p !}{\pi} \frac{e^{-C_{2} T^{\alpha-1}}}{C_{2} / T}\left(T^{\alpha}+T / C_{2}\right)^{p} .
\end{aligned}
$$

For $\alpha>1$, this decreases faster than any power of $T$. Combining these estimates implies the proposition.

According to Proposition 1 and equation (16), one now needs a good bound on the decay (in $n$ ) of the Green's function for complex energies in the vicinity of the spectrum. As shown by Damanik and Tcheremchantsev [DT], such bounds can be obtained for Jacobi matrices in a very efficient way in terms of the transfer matrices. Here we give a streamlined proof of this statement which also works for arbitrary Jacobi matrices (the kinetic part is not necessarily the discrete Lapalacian) and does not contain energy dependent constants as in [DT]. It will be convenient to first consider such bounds for the half-line problem, which is operator (44) on $\ell^{2}(\mathbb{N})$ with Dirichlet boundary conditions. This operator and its Green's function are denoted by $\hat{H}$ and $\hat{G}^{z}(n, m)$. 
Proposition 2 Set $\tau=\max \left\{\|t\|_{\infty}^{2}, 1,\left\|t^{-1}\right\|_{\infty}^{2}\right\}$ and $z=E+\frac{\imath}{T}$. One has the bounds

$$
\sum_{n>N}\left|\hat{G}^{z}(0, n)\right|^{2} \leq \frac{4 \tau^{3} T^{4}}{\max _{0 \leq n \leq N}\left\|\mathcal{T}^{z}(n, 0)\right\|^{2}}
$$

and, for $T \geq 1$,

$$
\sum_{|n|>N}\left|G^{z}(0, n)\right|^{2} \leq \frac{16 \tau^{4} T^{6}}{\max _{0 \leq|n| \leq N}\left\|\mathcal{T}^{z}(n, 0)\right\|^{2}}
$$

Proof. Let $\Gamma_{N}$ be the decoupling operator at $N$ defined by $\Gamma_{N}=t_{N+1}(|N\rangle\langle N+1|+| N+1\rangle\langle N|)$ and set $\hat{G}_{N}^{z}=\left(\hat{H}-z-\Gamma_{N}\right)^{-1}$ and $\hat{G}^{z}=(\hat{H}-z)^{-1}$. The resolvent identity reads

$$
\hat{G}^{z}=\hat{G}_{N}^{z}-\hat{G}_{N}^{z} \Gamma_{N} \hat{G}^{z} .
$$

Thus, with the notation $\hat{G}_{N}^{z}(n, m)=\left\langle n\left|\hat{G}_{N}^{z}\right| m\right\rangle$,

$$
\begin{aligned}
\sum_{n>N}\left|\hat{G}^{z}(0, n)\right|^{2} & =\sum_{n>N}\left|\hat{G}_{N}^{z} \Gamma_{N} \hat{G}^{z}(0, n)\right|^{2} \\
& =\left|\hat{G}_{N}^{z}(0, N) t_{N+1}\right|^{2} \sum_{n>N}\left|\hat{G}^{z}(N+1, n)\right|^{2} \leq\|t\|_{\infty}^{2} T^{2}\left|\hat{G}_{N}^{z}(0, N)\right|^{2}
\end{aligned}
$$

since $T^{-1}=\Im m(z)$ and $\left\|\hat{G}^{z}\right\| \leq T$. As the l.h.s. is decreasing in $N$, we therefore have

$$
\sum_{n>N}\left|\hat{G}^{z}(0, n)\right|^{2} \leq\|t\|_{\infty}^{2} T^{2} \min _{0 \leq n \leq N}\left|\hat{G}_{n}^{z}(0, n)\right|^{2}
$$

Now let $\Pi_{N}=\sum_{n=0}^{N}|n\rangle\langle n|$ be the projection on the states on the first $N+1$ sites and set $\hat{H}_{N}=\Pi_{N} H \Pi_{N}$. Then $\hat{G}_{N}^{z}(n, m), 0 \leq n, m \leq N$, are the matrix elements of the inverse of an $(N+1) \times(N+1)$ matrix $\hat{H}_{N}-z$. The matrix elements are closely linked to the transfer matrix

$$
\mathcal{T}^{z}(N+1,0)=\left(\begin{array}{ll}
a & b \\
c & d
\end{array}\right)
$$

since $a, b, c, d$ when multiplied by the $\prod_{n=1}^{N} t_{n}$, are the determinants of certain minors of $z-\hat{H}_{N}$. Namely by Cramer's rule (or, alternatively, by the Stieltjes continued fraction expansion and geometric resolvent identity) the following identities hold for $z \notin \sigma\left(\hat{H}_{N}\right)$ :

$$
\hat{G}_{N}^{z}(0,0)=\frac{1}{t_{0}^{2}} \frac{b}{a}, \quad \hat{G}_{N}^{z}(N, N)=-\frac{c}{a}, \quad \hat{G}_{N-1}^{z}(0,0)=\frac{1}{t_{0}^{2}} \frac{d}{c},
$$

and

$$
\hat{G}_{N}^{z}(0, N)=-\frac{1}{t_{0}} \frac{1}{a}, \quad \hat{G}_{N-1}^{z}(0, N-1)=-\frac{1}{t_{0} t_{N}} \frac{1}{c} .
$$

Therefore $|b| \leq t_{0}^{2} T|a|,|c| \leq T|a|$ and $|d| \leq t_{0}^{2} T|c|$. As the matrix norm is bounded by the Hilbert-Schmidt norm, it follows that

$$
\left\|\mathcal{T}^{z}(N+1,0)\right\|^{2} \leq \frac{4 T^{2} \tau^{2}}{\min \left\{\left|\hat{G}_{N}^{z}(0, N)\right|^{2},\left|\hat{G}_{N-1}^{z}(0, N-1)\right|^{2}\right\}} .
$$


By (17) this proves the first inequality. The second one follows from the first one (coupled with the same statement for the left half-line) by observing that the resolvent identity gives

$$
G^{z}(0, n)=\hat{G}^{z}(0, n)-G^{z}(0,-1) t_{0} \hat{G}^{z}(0, n) .
$$

Therefore $\left|G^{z}(0, n)\right| \leq\left(1+T t_{0}\right)\left|\hat{G}^{z}(0, n)\right|$ which implies the second bound.

\section{Logarithmic bounds in the localization phase}

In this section we provide the proof of Theorem 1 and hence suppose throughout that the stated hypothesis hold. The main idea is to use the given positivity of the Lyapunov exponent (7), combine it with the given uniform upper bound (6) in order to deduce good probabilistic estimates on the growth of the transfer matrices. This growth in turn allows to bound the Green's function due to Proposition 2 which then readily leads to the logarithmic upper bound on the moments.

Let us set $U=\left\{z \in \mathbb{C}\left|E_{0} \leq \Re e(z) \leq E_{1},\right| \Im m(z) \mid \leq 1\right\}$.

Lemma 3 For $z \in U$ and $N \in \mathbb{N}$, the set

$$
\Omega_{N}(z)=\left\{\omega \in \Omega \mid\left\|\mathcal{T}_{\omega}^{z}(N, 0)\right\|^{2} \geq e^{\gamma_{0} N}\right\}
$$

satisfies

$$
\mathbf{P}\left(\Omega_{N}(z)\right) \geq \frac{\gamma_{0}}{2 \gamma_{1}-\gamma_{0}}
$$

Proof. Let us set $P=\mathbf{P}\left(\Omega_{N}(z)\right)$. Due to (17), the subadditivity of the transfer-matrix cocycle and the bound (6), it follows that

$$
\gamma_{0} \leq \frac{1}{N} \mathbf{E} \log \left(\left\|\mathcal{T}_{\omega}^{z}(N, 0)\right\|\right) \leq(1-P) \frac{1}{2} \gamma_{0}+P \gamma_{1}
$$

with $\gamma_{1}$ defined by (6) using $U$ as above. This directly implies the result.

Lemma 4 Let $z \in U$ and $N \in \mathbb{N}$. Then there is a constant $C_{3}=C_{3}\left(\gamma_{0}, \gamma_{1}\right)$ such that the set

$$
\hat{\Omega}_{N}(z)=\left\{\omega \in \Omega \mid \max _{0 \leq n \leq N}\left\|\mathcal{T}_{\omega}^{z}(n, 0)\right\|^{2} \geq e^{C_{3} N^{\frac{1}{2}}}\right\}
$$

satisfies

$$
\mathbf{P}\left(\hat{\Omega}_{N}(z)\right) \geq 1-e^{-C_{3} N^{\frac{1}{2}}}
$$

Proof. Let us split $N$ into $\frac{N}{N_{0}}$ pieces of length $N_{0}$ (here and in the sections below, we suppose without giving further details that there is an integer number of pieces and that the boundary terms are treated separately). By the stationarity, on each piece $\left[j N_{0}+1,(j+1) N_{0}\right)$, Lemma 3 with $N=N_{0}$ applies. As the pieces are independent, we deduce

$$
\mathbf{P}\left(\left\{\omega \in \Omega \mid \max _{0 \leq j \leq N / N_{0}}\left\|\mathcal{T}_{\omega}^{z}\left((j+1) N_{0}, j N_{0}+1\right)\right\|^{2} \leq e^{\gamma_{0} N_{0}}\right\}\right) \leq\left(1-p_{0}\right)^{\frac{N}{N_{0}}}
$$


where $p_{0}=\gamma_{0} /\left(2 \gamma_{1}-\gamma_{0}\right)$. Furthermore $\mathcal{T}_{\omega}^{z}\left((j+1) N_{0}, j N_{0}+1\right)=\mathcal{T}_{\omega}^{z}\left((j+1) N_{0}, 0\right) \mathcal{T}_{\omega}^{z}\left(j N_{0}, 0\right)^{-1}$. As $A=B C$ implies either $\|B\| \geq\|A\|^{\frac{1}{2}}$ or $\|C\| \geq\|A\|^{\frac{1}{2}}$ for arbitrary matrices, and $\left\|A^{-1}\right\|=$ $\|A\|$ for $A \in \mathrm{SL}(2, \mathbb{C})$, it therefore follows that

$$
\mathbf{P}\left(\left\{\omega \in \Omega \mid \max _{0 \leq j \leq N / N_{0}}\left\|\mathcal{T}_{\omega}^{z}\left(j N_{0}, 0\right)\right\|^{2} \geq e^{\frac{1}{2} \gamma_{0} N_{0}}\right\}\right) \geq 1-\left(1-p_{0}\right)^{\frac{N}{N_{0}}} .
$$

Choosing $N_{0}=c N^{\frac{1}{2}}$ with adequate $c$ concludes the proof.

Since the above lemma applies equally well to negative integers $N$, it is a direct corollary of Lemma 4 and Proposition 2 that, for $T$ sufficiently large,

$$
\sum_{|n|>N} \mathbf{E}\left|G_{\omega}^{z}(0, n)\right|^{2} \leq 32 \tau^{4} T^{6} e^{-C_{3} N^{\frac{1}{2}}}, \quad z=E+\frac{\imath}{T} \in U
$$

The following lemma, holding for arbitrary ergodic families of Jacobi matrices, is useful for bounding a sum similar to the one in (18), but over $|n| \leq N$. Let $B_{\mu}(z)=\int \mu(d E)(z-E)^{-1}$ denote the Borel transform of a measure $\mu$.

Lemma 5 For any $E \in \mathbb{R}, T>0$ and $N \geq 1$, one has

$$
\sum_{0 \leq|n| \leq N} \frac{|n|^{q}}{\pi T} \mathbf{E}\left|G_{\omega}^{z}(0, n)\right|^{2} \leq \frac{N^{q}}{\pi} \Im m B_{\mathcal{N}}(z), \quad z=E+\frac{\imath}{T} .
$$

Furthermore, for any $E_{0}<E_{1}$,

$$
\sum_{0 \leq|n| \leq N}|n|^{q} \int_{E_{0}}^{E_{1}} \frac{d E}{\pi T} \mathbf{E}\left|G_{\omega}^{E+\frac{\imath}{T}}(0, n)\right|^{2} \leq N^{q}
$$

Proof. One has

$$
\begin{aligned}
\sum_{0 \leq|n|<N} \frac{|n|^{q}}{\pi T} \mathbf{E}\left|G_{\omega}^{z}(0, n)\right|^{2} & \leq N^{q} \sum_{n \in \mathbb{Z}} \frac{1}{\pi T} \mathbf{E}\left\langle 0\left|\frac{1}{H_{\omega}-E-\frac{l}{T}}\right| n\right\rangle\left\langle n\left|\frac{1}{H_{\omega}-E+\frac{l}{T}}\right| 0\right\rangle \\
& \leq N^{q} \mathbf{E} \frac{1}{\pi T}\left\langle 0\left|\frac{1}{\left(H_{\omega}-E\right)^{2}+\frac{1}{T^{2}}}\right| 0\right\rangle,
\end{aligned}
$$

which by the spectral theorem gives (19). Using

$$
\int_{E_{0}}^{E_{1}} \frac{d E}{\pi} \Im m B_{\mathcal{N}}\left(E+\imath T^{-1}\right) \leq \int_{\mathbb{R}} \frac{d E}{\pi T} \int_{\mathbb{R}} \mathcal{N}(d e) \frac{1}{(e-E)^{2}+\frac{1}{T^{2}}}=\int_{\mathbb{R}} \mathcal{N}(d e)=1
$$

the inequality (20) follows upon integrating (19).

Proof of Theorem 1, According to Proposition 1 it remains to bound $\mathbf{E} M_{T}^{q, 0, \alpha}\left(E_{0}, E_{1}\right)$ for $\alpha>1$. We further split this into two contributions:

$$
M_{T}(1)=\sum_{0 \leq|n| \leq(\log T)^{\beta}}|n|^{q} \int_{E_{0}}^{E_{1}} \frac{d E}{\pi T} \mathbf{E}\left|G_{\omega}^{E+\frac{\imath}{T}}(0, n)\right|^{2}
$$


and $M_{T}(2)$ corresponding to the sum over $(\log T)^{\beta}<|n| \leq T^{\alpha}$. The bound (20) with $N=$ $(\log T)^{\beta}$ immediately gives $M_{T}(1) \leq(\log T)^{\beta q}$. The second contribution can be bounded using (18):

$$
M_{T}(2) \leq \frac{64 \tau^{4} T^{5}}{\pi} \sum_{n \geq(\log T)^{\beta}} n^{q} e^{-C_{3} n^{\frac{1}{2}}},
$$

which is bounded by a constant $C(\beta, q)$ due to Lemma 2 as long as $\beta>2$.

This above proof applies equally well to the half-line problem with arbitrary boundary condition, the only difference being that the IDS $\mathcal{N}$ has to be replaced by the $\mathbf{E}\left(\mu_{\omega}(d E)\right)$ where $\mu_{\omega}$ is the spectral measure of $|0\rangle$ and $\hat{H}$.

\section{$5 \quad$ Probabilistic estimates near a critical energy}

In this section, we first derive more quantitative versions of Lemmata 3 and 4 by replacing the input (6) and (7) by the estimates (11) and (12). However, these estimates are not sufficient for the proof of Theorem 3. In fact, one can further improve the Lemmata by replacing the uniform upper bound (11) by a probabilistic one, deduced from a large deviation estimate from [JSS] recalled below and showing that the transfer matrices grow no more than given by the Lyapunov exponent with high probability. For sake of notational simplicity, we suppose that $E_{c}=0$. Furthermore, according to (12) and the Thouless formula we may choose positive $d<D$ and $\epsilon_{0}$ such that

$$
\gamma(z) \geq d \epsilon^{2}, \quad \text { for } z=\epsilon+\imath \delta \text { with }|\epsilon|<\epsilon_{0} .
$$

In order to further simplify notation, we also assume that $\delta, \epsilon>0$ even though all estimates hold with $|\delta|$ and $|\epsilon|$.

Lemma 6 For $z=\epsilon+\imath \delta$ with $\delta \leq \epsilon<\epsilon_{0}$ introduce the set

$$
\Omega_{N}(z)=\left\{\omega \in \Omega \mid\left\|\mathcal{T}_{\omega}^{z}(N, 0)\right\|^{2} \geq e^{d \epsilon^{2} N}\right\}
$$

Then

$$
\mathbf{P}\left(\Omega_{N}(z)\right) \geq c_{1} \epsilon
$$

Proof. This is an immediate corollary of Lemma 3 with $c_{1}=d /\left(2 c_{0}\right)$, where $c_{0}$ is introduced in (11).

Lemma 7 Let $z=\epsilon+\imath \delta$ with $\delta \leq \epsilon<\epsilon_{0}$. Then there exists a constant $c_{2}$ such that the set

$$
\hat{\Omega}_{N}(z)=\left\{\omega \in \Omega \mid \max _{0 \leq n \leq N}\left\|\mathcal{T}_{\omega}^{z}(n, 0)\right\|^{2} \geq e^{\epsilon^{3} N}\right\}
$$

satisfies

$$
\mathbf{P}\left(\hat{\Omega}_{N}(z)\right) \geq 1-e^{-c_{2} N}
$$


Proof. Let us split $N$ into $\frac{N}{N_{0}}$ pieces of length $N_{0}$ and follow the proof of Lemma 4 invoking Lemma 6 instead of Lemma 3 , giving

$$
\mathbf{P}\left(\left\{\omega \in \Omega \mid \max _{0 \leq n \leq N}\left\|\mathcal{T}_{\omega}^{z}(n, 0)\right\|^{2} \geq e^{\frac{1}{2} d \epsilon^{2} N_{0}}\right\}\right) \geq 1-\left(1-c_{1} \epsilon\right)^{\frac{N}{N_{0}}} \geq 1-e^{-c_{1} \epsilon \frac{N}{N_{0}}} .
$$

Choosing $N_{0}=\frac{2 \epsilon}{d}$ shows that one may take $c_{2}=c_{1} d / 2$.

Certainly other choices of $N_{0}$ are possible in the previous proof, but the present one leading to Lemma 7 implies the following estimate, which is sufficient in order to deal with one of the terms in the next section (a boundary term of energies close to $\epsilon_{0}$ ).

Corollary 1 Let $z=\epsilon+\imath \delta$ with $\delta \leq \epsilon<\epsilon_{0}$. There is a constant $c_{3}$ such that

$$
\mathbf{E}\left(\frac{1}{\max _{0 \leq|n| \leq N}\left\|\mathcal{T}^{z}(n, 0)\right\|^{2}}\right) \leq e^{-c_{3} \epsilon^{3} N} .
$$

Now we turn to the refined statements and start by recalling the following

Theorem 4 [JSS Suppose that $\mathbf{E}\left(e^{2 \imath \eta_{\sigma}}\right) \neq 1$ and $0<\alpha \leq \frac{1}{2}$. Then there exist constants $c_{4}, c_{5}, c_{6}, c_{7}$ such that the set

$$
\Omega_{N}^{\alpha}=\left\{\omega \in \Omega\left|\max _{0 \leq n, m \leq N}\left\|\mathcal{T}_{\omega}^{\epsilon+\imath \delta}(n, m)\right\| \leq e^{c_{4}} \forall\right| \delta\left|\leq c_{5} N^{-1},\right| \epsilon \mid \leq N^{-\frac{1}{2}-\alpha}\right\},
$$

satisfies

$$
\mathbf{P}\left(\Omega_{N}^{\alpha}\right) \geq 1-c_{6} e^{-c_{7} N^{\alpha}} .
$$

For a fixed energy $z$, this can be extended to length scales $N$ beyond the localization length (inverse Lyapunov exponent).

Lemma 8 For $z=\epsilon+\imath \delta$ with $\delta \leq c_{5} \epsilon^{2}$ and $\epsilon \geq N^{-\frac{1}{2}-\alpha}$, the set

$$
\Omega_{N}^{\alpha}(z)=\left\{\omega \in \Omega \mid \begin{array}{l|l}
\max _{0 \leq n, m \leq N}\left\|\mathcal{T}_{\omega}^{z}(n, m)\right\| \leq e^{c_{4} N \epsilon^{2(1-2 \alpha)}}
\end{array}\right\},
$$

satisfies

$$
\mathbf{P}\left(\Omega_{N}^{\alpha}(z)\right) \geq 1-c_{6} N \epsilon e^{-c_{7} \epsilon^{-\alpha}} .
$$

Proof. We split $N$ into $\frac{N}{N_{0}}$ pieces of length $N_{0}=\epsilon^{-\frac{2}{2 \alpha+1}}$. The condition $\epsilon \geq N^{-\frac{1}{2}-\alpha}$ insures that $N_{0} \leq N$. By the stationarity, on each piece we may apply Theorem 4 (with $N_{0}$ instead of $N$ ) because $\epsilon \leq N_{0}^{-\frac{1}{2}-\alpha}$. For the $j$ th piece, denote the set appearing in Theorem 4 by $\Omega_{N}^{\alpha, j}$. For any $\omega \in \cap_{j=1, \ldots, N} \Omega_{0} \Omega_{N}^{\alpha, j}$ one then has for $\delta \leq c_{5} N_{0}^{-1}$ (and hence also $\delta \leq c_{5} \epsilon^{2}$ ) the estimate

$$
\max _{0 \leq n, m \leq N}\left\|\mathcal{T}_{\omega}^{z}(n, m)\right\| \leq e^{c_{4} \frac{N}{N_{0}}} \leq e^{c_{4} N \epsilon^{2(1-2 \alpha)}} .
$$


Therefore $\cap_{j=1, \ldots, \frac{N}{N_{0}}} \Omega_{N}^{\alpha, j} \subset \Omega_{N}^{\alpha}(z)$. We hence deduce from Theorem 4 that

$$
\mathbf{P}\left(\Omega_{N}^{\alpha}(z)^{c}\right) \leq c_{6} \frac{N}{N_{0}} e^{-c_{7} N_{0}^{\alpha}} \leq c_{6} N \epsilon e^{-c_{7} \epsilon^{-\alpha}},
$$

which is precisely the statement of the lemma.

This last lemma can now be used in order to improve Lemma 6 in the range $\delta<c \epsilon^{2}$.

Lemma 9 Let $z=\epsilon+\imath \delta$ with $\delta \leq c_{5} \epsilon^{2}$ and $N^{-\frac{1}{2}-\alpha} \leq \epsilon \leq N^{-\alpha}, 0<\alpha \leq \frac{1}{2}$. Then the set $\Omega_{N}(z)$ defined in (22) satisfies for some constant $c_{8}$

$$
\mathbf{P}\left(\Omega_{N}(z)\right) \geq c_{8} \epsilon^{4 \alpha} .
$$

Proof. We argue as in the proof of Lemma 3. Let us set again $P=\mathbf{P}\left(\Omega_{N}(z)\right)$, and estimate separately the contribution from the complement of $\Omega_{N}(z), \Omega_{N}^{\alpha}(z)$ and it's complement. Due to (21), Lemma 8 and the a priori bound (11) (used on the complement of $\Omega_{N}^{\alpha}(z)$ ), it follows that

$$
d \epsilon^{2} \leq(1-P) \frac{1}{2} d \epsilon^{2}+P c_{4} \epsilon^{2(1-2 \alpha)}+2 c_{0} c_{6} N \epsilon^{2} e^{-c_{7} \epsilon^{-\alpha}} .
$$

Hence

$$
P \geq \epsilon^{4 \alpha} \frac{d-4 c_{0} c_{6} N e^{-c_{7} \epsilon^{-\alpha}}}{2 c_{4}-d \epsilon^{4 \alpha}} .
$$

The hypothesis $\epsilon \leq N^{-\alpha}$ implies the result (it would be enough to assume $\epsilon \leq \log (N)^{p}$ for some $p)$.

As the final preparatory step for the next section, we improve Lemma 7 in the range $\delta<c \epsilon^{2}$, by invoking Lemma 9 in its proof.

Lemma 10 Let $z=\epsilon+\imath \delta$ with $\delta \leq c_{5} \epsilon^{2}$ and $N^{-\frac{1}{2}-\alpha} \leq \epsilon \leq N^{-\alpha}, 0<\alpha \leq \frac{1}{2}$. Then the set

$$
\hat{\Omega}_{N}^{\alpha}(z)=\left\{\begin{array}{l|l|l|}
\omega \in \Omega & \max _{0 \leq n \leq N}\left\|\mathcal{T}_{\omega}^{z}(n, 0)\right\|^{2} \geq e^{N^{1-\alpha} \epsilon^{2(1+2 \alpha)}}
\end{array}\right\}
$$

satisfies for some constant $c_{9}$

$$
\mathbf{P}\left(\hat{\Omega}_{N}^{\alpha}(z)\right) \geq 1-e^{-c_{9} N^{\alpha}} .
$$

Proof. Splitting $N$ into $\frac{N}{N_{0}}$ pieces of length $N_{0}$ and arguing exactly as in Lemma 4 invoking Lemma 9 instead of Lemma 3, we obtain

$$
\mathbf{P}\left(\left\{\omega \in \Omega \mid \max _{0 \leq n \leq N}\left\|\mathcal{T}_{\omega}^{z}(n, 0)\right\|^{2} \geq e^{\frac{1}{2} d \epsilon^{2} N_{0}}\right\}\right) \geq 1-\left(1-c_{8} \epsilon^{4 \alpha}\right)^{\frac{N}{N_{0}}}
$$

Choosing $N_{0}=\left(2 N^{1-\alpha} \epsilon^{4 \alpha}\right) / d$ shows that one may take $c_{9}=c_{8} d / 2$.

Lemma 10 implies the following estimate, which is the main result of this section and will be used in the next one.

Corollary 2 Let $z=\epsilon+\imath \delta$. If $\delta \leq c_{5} \epsilon^{2}$ and $N^{-\frac{1}{2}-\alpha} \leq \epsilon \leq N^{-\alpha}, 0<\alpha \leq \frac{1}{2}$, one has

$$
\mathbf{E}\left(\frac{1}{\max _{0 \leq|n| \leq N}\left\|\mathcal{T}^{z}(n, 0)\right\|^{2}}\right) \leq e^{-N^{1-\alpha} \epsilon^{2(1+2 \alpha)}}+e^{-c_{9} N^{\alpha}} .
$$




\section{Proof of upper bound for random polymer models}

In this section we complete the proof of Theorem 3. For this purpose, we follow the strategy discussed in Section 3 and consider $M_{T}^{q, \alpha_{0}, \alpha_{1}}\left(E_{0}, E_{1}\right)$ defined as in (16), but with a disorder average E. By Proposition 1 it is sufficient to bound $M_{T}^{q, 0,1+\alpha}\left(E_{0}, E_{1}\right)$ for $\alpha>0$ if $\left(E_{0}, E_{1}\right)$ contains the spectrum. Moreover, energies bounded away from critical energies have a strictly positive Lyapunov exponent [BG]. By the results of Section 4 , these energies hence lead at most to logarithmic growth in time, and therefore give no contribution to the diffusion exponents $\beta_{q}$. Thus we are left to deal with energy intervals around the critical energies. All of them are treated the same way, so we focus on one of them. We suppose that $E_{c}=0$ and consider only the energy interval $\left[0, \epsilon_{0}\right]$ with $\epsilon_{0}$ chosen as in (21); the other side $\left[-\epsilon_{0}, 0\right]$ is again treated similarly. Furthermore we split the contribution as follows

$$
M_{T}^{q, 0,1+\alpha}\left(0, \epsilon_{0}\right)=M_{T}^{q, 0,1+\alpha}\left(0, T^{-\eta}\right)+M_{T}^{q, 0,1+\alpha}\left(T^{-\eta}, T^{-\alpha}\right)+M_{T}^{q, 0,1+\alpha}\left(T^{-\alpha}, \epsilon_{0}\right),
$$

where $\eta=\min \left\{q, \frac{1}{2}\right\}$. This is a good choice due to the following lemma, showing that the contribution $M_{T}^{q, 0,1+\alpha}\left(0, T^{-\eta}\right)$ is bounded by the diffusion exponent as given in Theorem 3 ,

Lemma 11 For some constant $C_{1}$, one has

$$
M_{T}^{q, 0,1+\alpha}\left(0, T^{-\eta}\right) \leq C_{1} T^{q-\eta+\alpha q} .
$$

Proof. By (19) we have

$$
M_{T}^{q, 0,1+\alpha}\left(0, T^{-\eta}\right) \leq T^{q(1+\alpha)} \int_{0}^{T^{-\eta}} d \epsilon \Im m B_{\mathcal{N}}\left(\epsilon+\imath T^{-1}\right) .
$$

The estimate now follows from (13) and Proposition 3 in the appendix, with $\epsilon_{0}=T^{-\eta}$.

Next let us consider the boundary term $M_{T}^{q, 0,1+\alpha}\left(T^{-\alpha}, \epsilon_{0}\right)$.

Lemma 12 For some constant $C_{2}=C_{2}(\alpha)$, one has

$$
M_{T}^{q, 0,1+\alpha}\left(T^{-\alpha}, \epsilon_{0}\right) \leq T^{4 \alpha q}+C_{2}
$$

Proof. Let us split the sum over $n$ appearing in the definition of $M_{T}^{q, 0,1+\alpha}\left(T^{-\alpha}, \epsilon_{0}\right)$ into one over $|n| \leq T^{4 \alpha}$ and the other over $|n|>T^{4 \alpha}$. The first one can be bounded by $T^{4 \alpha q}$ using (20). In the second one we apply Proposition 2 combined with Corollary 1 in order to bound the Green's function. This shows $M_{T}^{q, 0,1+\alpha}\left(T^{-\alpha}, \epsilon_{0}\right)$ is bounded above by

$$
T^{4 \alpha q}+\int_{T^{-\alpha}}^{\epsilon_{0}} \frac{d \epsilon}{\pi T} \sum_{|n|>T^{4 \alpha}}|n|^{q} 16 \tau^{4} T^{6} e^{-c_{3} \epsilon^{3}|n|} .
$$

In the second term, the sum over $n$ is bounded by Lemma 2. As $\epsilon>T^{-\alpha}$, it follows that the second term is bounded by $C T^{p} e^{-c_{3} T^{\alpha}}$ for some $C, p>0$. Hence this term gives the second contribution in the bound. 
Lemma 13 For $\eta=\min \left\{q, \frac{1}{2}\right\}$ and some constants $C_{3}=C_{3}(\alpha)$ and $C_{4}$, one has

$$
M_{T}^{q, 0, \infty}\left(T^{-\eta}, T^{-\alpha}\right) \leq C_{3}+C_{4} T^{6 q \alpha} \cdot\left\{\begin{array}{cc}
T^{q-\frac{1}{2}} & q \geq \frac{1}{2} \\
T^{\alpha(2 q-1)} & q \leq \frac{1}{2} .
\end{array}\right.
$$

Proof. We first split $M_{T}^{q, 0, \infty}\left(T^{-\eta}, T^{-\alpha}\right)$ into two contributions $M_{T}(1)$ and $M_{T}(2)$, the first containing all the summands with $|n|$ smaller than the (energy dependent) localization length:

$$
M_{T}(1)=\int_{T^{-\eta}}^{T^{\alpha}} \frac{d \epsilon}{\pi T} \sum_{1 \leq|n| \leq \epsilon^{-2} T^{4 \alpha}}|n|^{q} \mathbf{E}\left|G_{\omega}^{\epsilon+\frac{\imath}{T}}(0, n)\right|^{2}
$$

and the second $M_{T}(2)$ containing the sum over $|n|>\epsilon^{-2} T^{4 \alpha}$ corresponding to the summands beyond the localization length. $M_{T}(1)$ is bounded using (19):

$$
M_{T}(1) \leq T^{4 \alpha q} \int \mathcal{N}(d E) \int_{T^{-\eta}}^{T^{-\alpha}} \frac{d \epsilon}{\pi T} \epsilon^{-2 q} \frac{1}{(E-\epsilon)^{2}+T^{-2}}
$$

In order to bound the factor $\epsilon^{-2 q}$, let us split the integral over $\epsilon$ into $\frac{\eta-\alpha}{\alpha}$ pieces:

$$
\begin{aligned}
M_{T}(1) & \leq T^{4 \alpha q} \sum_{j=1}^{\frac{\eta-\alpha}{\alpha}} T^{2 q(\eta-(j-1) \alpha)} \int \mathcal{N}(d E) \int_{T^{-\eta+(j-1) \alpha}}^{T^{-\eta+j \alpha}} \frac{d \epsilon}{\pi T} \frac{1}{(E-\epsilon)^{2}+T^{-2}} \\
& =T^{4 \alpha q} \sum_{j=1}^{\frac{\eta-\alpha}{\alpha}} T^{2 q(\eta-(j-1) \alpha)} \int_{T^{-\eta+(j-1) \alpha}}^{T^{-\eta+j \alpha}} d \epsilon \Im m B_{\mathcal{N}}\left(\epsilon+\imath T^{-1}\right) .
\end{aligned}
$$

Using Proposition 3 we obtain

$$
M_{T}(1) \leq C T^{4 \alpha q} \sum_{j=1}^{\frac{\eta-\alpha}{\alpha}} T^{2 q(\eta-(j-1) \alpha)} T^{-\eta+j \alpha}=C T^{6 q \alpha} T^{(2 q-1) \eta} \sum_{j=1}^{\frac{\eta-\alpha}{\alpha}} T^{(1-2 q) \alpha j}
$$

For $q \geq 1 / 2$, we use the bound $T^{(1-2 q) \alpha j} \leq 1$ showing that the sum is bounded by $\frac{\eta-\alpha}{\alpha}$. For $q \leq 1 / 2$ we bound each summand by $T^{(1-2 q) \alpha j} \leq T^{(1-2 q)(\eta-\alpha)}$. This gives the second contribution in the lemma.

It remains to show that $M_{T}(2) \leq C_{3}$. Due to Proposition 2 and Corollary 2 ,

$$
M_{T}(2) \leq \int_{T^{-\eta}}^{T^{-\alpha}} \frac{d \epsilon}{\pi T} \sum_{|n|>\epsilon^{-2} T^{4 \alpha}}|n|^{q} 16 \tau^{4} T^{6}\left(e^{-\epsilon^{2(1+2 \alpha)}|n|^{1-\alpha}}+e^{-c_{9}|n|^{\alpha}}\right) .
$$

Using Lemma 2 it is now elementary to bound $M_{T}(2)$ by a constant.

Combining Lemmata 11, 12 and 13, and recalling that $\alpha$ can be taken arbitrary close to 0 , proves Theorem 3 . 


\section{Appendix: estimates on the Borel transform}

In Section 6 we used well-known estimates on the Borel transform $B_{\mu}(z)=\int \mu(d E)(z-E)^{-1}$ of a measure $\mu$. For sake of completeness we provide a short proof.

Proposition 3 If a measure $\mu$ satisfies at some $E$ the bound $\mu([E-\epsilon, E+\epsilon])<C \epsilon$ for all $\epsilon>0$, then for any finite positive $\delta$ and $\epsilon_{0}$

$$
\Im m B_{\mu}(E+\imath \delta)<\frac{\pi}{2} C, \quad \int_{0}^{\epsilon_{0}} d \epsilon \Im m B_{\mu}(E+\epsilon+\imath \delta)<\pi^{2} C \epsilon_{0} .
$$

Proof. One has, uniformly in $\delta$,

$$
\begin{aligned}
\Im m B_{\mu}(E+\imath \delta) & =\int \mu(d e) \frac{\delta}{(e-E)^{2}+\delta^{2}}=\delta \int_{0}^{\frac{1}{\delta^{2}}} d t \mu\left(\left\{e \in \mathbb{R}|| e-E \mid<\sqrt{\frac{1}{t}-\delta^{2}}\right\}\right) \\
& <C \delta \int_{0}^{\frac{1}{\delta^{2}}} d t \sqrt{\frac{1}{t}-\delta^{2}}<C \int_{0}^{\infty} d x \frac{\sqrt{x}}{(x+1)^{2}},
\end{aligned}
$$

which gives the first inequality. For the second one, let us bound the indicator function $\chi_{\left[-\epsilon_{0}, \epsilon_{0}\right]}(\epsilon)$ above by $\frac{2 \epsilon_{0}^{2}}{\epsilon^{2}+\epsilon_{0}^{2}}$. Then one can use the stability of Cauchy distribution to obtain

$$
\begin{aligned}
\int_{0}^{\epsilon_{0}} d \epsilon \Im m B_{\mu}(E+\epsilon+\imath \delta) & <\int d \epsilon \frac{2 \epsilon_{0}^{2}}{\epsilon^{2}+\epsilon_{0}^{2}} \int \mu(d e) \frac{\delta}{(E+\epsilon-e)^{2}+\delta^{2}} \\
& =2 \pi \epsilon_{0} \int \mu(d e) \frac{\delta+\epsilon_{0}}{(E-e)^{2}+\left(\delta+\epsilon_{0}\right)^{2}}, \\
& =2 \pi \epsilon_{0} \Im m B_{\mu}\left(E+\imath\left(\delta+\epsilon_{0}\right)\right),
\end{aligned}
$$

and thus the second inequality follows from the first one.

\section{References}

[AM] M. Aizenman, S. Molchanov, Localization at Large Disorder and at Extreme Energies: an Elementary Derivation, Commun. Math. Phys. 157, 245-278 (1993).

[BGT] J.M. Barbaroux, F. Germinet, S. Tcheremchantsev, Fractal Dimensions and the Phenomenon of Intermittency in Quantum Dynamics, Duke Math. J. 110, 161-193 (2001).

[BS] J.-M. Barbaroux, H. Schulz-Baldes, Anomalous transport in presence of self-similar spectra, Annales I.H.P. Phys. théo. 71, 539-559 (1999).

[BeS] J. Bellissard, H. Schulz-Baldes, Subdiffusive quantum transport for 3D-Hamiltonians with absolutely continuous spectra, J. Stat. Phys. 99, 587-594 (2000).

[BG] S. de Bièvre, F. Germinet, Dynamical Localization for the Random Dimer Schrödinger Operator, J. Stat. Phys. 98, 1135-1148 (2000). 
[BL] P. Bougerol, J. Lacroix, Products of Random Matrices with Applications to Schrödinger Operators, (Birkhäuser, Boston, 1985).

[Bov] A. Bovier, Perturbation theory for the random dimer model, J. Phys. A: Math. Gen. 25, 1021-1029 (1992).

[BLS] J. Breuer, Y. Last, Y. Strauss, Upper bounds on the dynamical spreading of wavepackets, in preparation.

[CKM] R. Carmona, A. Klein, F. Martinelli, Anderson localization for Bernoulli and other singular potentials, Commun. Math. Phys. 108, 41-66 (1987).

[Com] J.-M. Combes, Connections between quantum dynamics and spectral properties of timeevolution operators, in Differential Equations with Applications to Mathematical Physics, Ames W.F., Harell E.M., Herod J.V. Eds, Academic Press, Boston (1993).

[CM] J.-M. Combes, G. Mantica, Fractal dimensions and quantum evolution associated with sparse potential Jacobi matrices, Proceedings of the Bologna APTEX international conference, World Scientific. Ser. Concr. Appl. Math. 1, 107-123 (2001).

[DSS] D. Damanik, R. Sims, G. Stolz, Localization for discrete one-dimensional random word models, J. Funct. Anal. 208, 423-445 (2004).

[DT] D. Damanik, S. Tcheremchantsev, Upper bounds in quantum dynamics, preprint 2005.

[DJLS] R. del Rio, S. Jitomirskaya, Y. Last, B. Simon, Operators with singular continuous spectrum: IV, Hausdorff dimension, rank-one perturbations and localization, J. d'Analyse Math. 69, 153-200 (1996).

[DMS] R. del Rio, N. Makarov, B. Simon, Operators with singular continuous spectrum: II, Rank one operators, Commun. Math. Phys. 165, 59-67 (1994).

[DWP] D. H. Dunlap, H.-L. Wu, P. W. Phillips, Absence of Localization in Random-Dimer Model, Phys. Rev. Lett. 65, 88-91 (1990).

[Gor] A. Gordon, Pure point spectrum under 1-parameter perturbations and instability of Anderson localization, Commun. Math. Phys. 164, 489-505 (1994).

[Gua] I. Guarneri, Spectral properties of quantum diffusion on discrete lattices, Europhys. Lett., 10, 95-100, (1989); On an estimate concerning quantum diffusion in the presence of a fractal spectrum, Europhys. Lett., 21, 729-733, (1993).

[GS1] I. Guarneri, H. Schulz-Baldes, Upper bounds for quantum dynamics governed by Jacobi matrices with self-similar spectra, Rev. Math. Phys. 11, 1249-1268 (1999).

[GS2] I. Guarneri, H. Schulz-Baldes, Lower bounds on wave packet propagation by packing dimensions of spectral measures, Elect. J. Math. Phys. 5, (1999). 
[GS3] I. Guarneri, H. Schulz-Baldes, Intermittent lower bound on quantum diffusion, Lett. Math. Phys. 49, 317-324 (1999).

[Jit] S. Jitomirskaya, Ergodic Schrödinger operators (on one foot). preprint 2006, to appear in Barry Simon Festschrift.

[JL] S. Jitomirskaya, Y. Last, Power-Law subordinacy and singular spectra, I. Half line operators. Acta Math. 183 , no. 2, 171-189 (1999).

[JSS] S. Jitomirskaya, H. Schulz-Baldes, G. Stolz, Delocalization in random polymer chains, Commun. Math. Phys. 233, 27-48 (2003).

[KKL] R. Killip, A. Kiselev, Y. Last, Dynamical upper bounds on wavepacket spreading, Amer. J. Math. 125, 1165-1198 (2003).

[KL] A. Kiselev, Y. Last, Solutions, spectrum, and dynamics for Schrödinger operators on infinite domains, Duke Math. J. 102, 125-150 (2000).

[Las] Y. Last, Quantum Dynamics and decomposition of singular continuous spectra, J. Funct. Anal. 142, 402-445 (1996).

$[\mathrm{PF}]$ L. Pastur, A. Figotin, Spectra of Random and Almost-Periodic Operators, (Springer, Berlin, 1992).

[SSS] R. Schrader, H. Schulz-Baldes, A. Sedrakyan, Perturbative test of single parameter scaling for $1 D$ random media, Annales H. Poincare 5, 1159-1180 (2004).

[Sch] H. Schulz-Baldes, Lyapunov exponents at anomalies of $\mathrm{SL}(2, \mathbb{R})$ actions, to appear in Operator Theory: Advances and Applications, (Birkhäuser, Basel, 2006).

[Tch] S. Tcheremchantsev, Dynamical analysis of Schrödinger operators with growing sparse potentials, Commun. Math. Phys. 253, 221-252 (2005). 